\title{
A theory of sequential group reciprocity
}

\author{
Alejandro T. Moreno-Okuno ${ }^{1}$ (D) Alejandro Mosiño ${ }^{1}$
}

Received: 9 November 2015/Revised: 13 July 2017/ Accepted: 19 July 2017 /

Published online: 22 August 2017

(C) The Author(s) 2017. This article is an open access publication

\begin{abstract}
Games that appear to be independent, involving none of the same players, may be related by emotions of reciprocity between the members of the same groups. In the real world, individuals are members of groups and want to reward or punish those groups whose members have been kind or unkind to members of their own. In this paper, we extend Dufwenberg and Kirchsteiger's model of sequential reciprocity (Games Econ Behav 47(2):268-298, 2004) to groups of individuals and define a new "sequential group reciprocity equilibrium" for which we prove its existence. We study the case of two games with two players in each game, where each player belongs to the same group as a player in the other game. We show that when the payoffs of one game are much higher than the payoffs of the other, the outcome of the game with higher payoffs determines the outcome of the other game. We also find that when the payoffs are very asymmetric, the outcome where the sum of the payoffs is maximized is a sequential group reciprocity equilibrium.
\end{abstract}

Keywords Fairness · Groups · Psychological games · Game theory

JEL classification A12 - D63 - C70

Alejandro T. Moreno-Okuno

atatsuo@hotmail.com

1 Universidad de Guanajuato, Guanajuato, Mexico 


\section{Introduction}

Usually, individuals want to pay kind acts with kindness and bad acts with unkindness. This is known as fairness or reciprocity. Most models in economics tend to ignore fairness or any other emotion despite its profound impact on economic activity. For example, Rabin (1993) showed that consumers may not consume a product (even if it is in their interest to do it) if they think its price is unfair. ${ }^{1}$ Akerlof (1982) argues that workers repay high wages by giving a high effort in their jobs. In the negative side, Krueger and Mas (2004) argue that after the management of Firestone demanded concessions from the workers and hired replacement workers during a strike, the workers responded by sabotaging the products which resulted in 271 fatalities.

In his seminal paper, Rabin (1993) introduced fairness to game theory by modeling how if one player believes that her opponent is sacrificing her own material payoffs to help her, then she may be willing to sacrifice her own material payoffs as well to help her opponent in return; and if one player believes that her opponent is treating her badly, she may sacrifice her own material payoff to treat her opponent badly. As an example, Rabin shows how in the Prisoners' Dilemma this allows for a "fairness equilibrium" (FE from now on) in which both players play "cooperate" . Rabin's model is defined for two players in static games. Dufwenberg and Kirchsteiger (2004) extended Rabin's model to dynamic games for more players with their solution concept of "sequential reciprocity equilibrium" (SRE from now on).

However, these models assume that players do not care about the outcomes of games in which they are not involved. In the real world individuals form emotions of fairness based on the treatment of others and these emotions can affect the outcome of their own games. For example, a mother may be nicer to someone who is nice to her daughter. And in countless interethnic conflicts, individuals from each of the groups involved have targeted innocent members of the other group to avenge previous offenses. Less tragically, individuals sometimes buy products at a higher price or of subpar quality solely because those products come from firms owned by their fellow countrymen or by members of their own ethnic group.

The aim of this paper is to analyze the relationship between the outcome of seemingly independent games (those games in which the players of one game are different to the players of the others), stemming from the emotions of fairness and reciprocity. For this, we extend Dufwenberg and Kirchsteiger (2004)'s model of reciprocity, in which individuals want to reciprocate the kindness or unkindness of other individuals, to a more comprehensive model that incorporates a sense of fairness that depends on the payoffs of other members of our own groups.

Other economists have developed other models of reciprocity. Falk and Fischbacher (2006), Levine (1998), Fehr and Schmidt (1999), and Bolton and Ockenfels (2000) are some examples. However, we focus in Dufwenberg and

\footnotetext{
${ }^{1}$ Kahneman et al. (1986) showed that individuals evaluate the fairness of an increase in the price of a product as fair or unfair, depending on the motives of the increase. Individuals consider an increase in the price of a product due to a higher demand as unfair and consider an increase in the price of a product due to an increase in costs as fair.
} 
Kirchsteiger's model, given that we believe their approach of reciprocity based on intentions is essential to represent the idea of group reciprocity.

The contribution of this article is to extend the concept of reciprocity with the following three stylized facts:

1. Individuals want to increase the material payoffs of those individuals who belong to the same groups as them and are willing to sacrifice their own material payoffs in order to do it.

2. Individuals treat more kindly those individuals who have been kind to other individuals who are close to them and individuals treat more unkindly those individuals who have been kind to other individuals who are close to them.

3. The acts of kindness or unkindness of some members of a group can create sympathy or resentment towards all the members of their group and a desire for reciprocity towards each of these, even those who played no part in the original acts.

We develop a game theoretic framework that incorporates these facts. In Sect. 2, we present evidence that support the stylized facts of our model. We review both empirical and experimental evidence.

In Sect. 3, we introduce our model by extending Dufwenberg and Kirchsteiger (2004)'s model of sequential reciprocity to groups of individuals. We define a utility function that represents all three stylized facts mentioned above and define a new solution concept which we call "sequential group reciprocity equilibria".

In Sect. 3.1, we probe the existence of our solution concept in Theorem 1. Although the proof is in "Appendix", we give a brief explanation of the proof. In Sect. 3.1.1 we mention the differences between our model and the FE and SRE.

In Sect. 3.2, we develop an example to show the relation that the outcome of one game has over the outcome of other games, due to emotions of group reciprocity. To show the interaction between groups of individuals, we work with the easiest case: two games of two players and two groups with two members. One of the members of each group play in each game. We get the following basic results:

First, as the material payoffs of one game grow arbitrarily large, the outcome of that game dominates the emotions of reciprocity of the players who play in the other game. In the sequential group reciprocity equilibria, if the outcome of the game with arbitrarily large payoffs is positive and both players are kind, the emotions of the players in the other game are going to be positive and they will be kind also in their own game. In the sequential group reciprocity equilibria, if the outcome of the game with arbitrarily large payoffs is negative and both players are unkind, the emotions of the players in the other game are going to be negative and they will be unkind also in their own game.

Second, in the sequential group reciprocity equilibria, when the material payoffs of one game become arbitrarily small, the sign of outcome of that game is the same as the sign of the outcome of the other game players in the game in which payoffs are small are kind only if players in the other game are kind and unkind if players in the other game are unkind. These two results are based on the same idea. If the stakes in one game are much higher in one game in relation to the other, the outcome of that game is going to dominate the emotions of the players also in other games. 
Third, if the material payoffs of both games are sufficiently asymmetric (i.e., individuals can greatly reward or punish their opposing players with little cost to themselves), the outcome in which the sum of the material payoffs is maximized is a sequential group reciprocity equilibrium. This result is very optimistic as it concludes that in the outcome where the sum of the material payoffs is being maximized, nobody wants to deviate (even if they can increase their own material payoffs by doing it). The reason is that in this case, the emotional payoffs is positive, in fact very positive, and even the players who can increase their material payoffs by deviating, prefer to maximize their emotional payoffs instead and being kind (as their partners are being treated nice, in fact, very nice).

Fourth, in the case where one game is played first and then the other, and the material payoffs of the games are asymmetric enough, we find that the sequential group reciprocity equilibrium is that in which the outcome in which the sum of the material payoffs of the players is maximized. The reason for this is that the player in the first period than can be very kind or unkind, wants to be nice to her opposite in order for her own partner to be treated nicely in the second period.

Finally, in Sect. 4, we conclude and discuss possible extensions.

\section{Evidence}

There is evidence that individuals often treat better other members of their own groups. Tajfel et al. (1971) show that individuals favor members of their own group when distributing rewards and penalties in situations in which irrelevant classifications distinguished between the in-group and the out-group. Ayres and Siegelman (1995) show that black buyers of new cars are offered higher prices than white buyers in dealerships in the Chicago area. Zussman (2013) finds that Arab buyers and sellers in the Israeli online market for used cars are discriminated against. Price and Wolfers (2010) find that referees in the NBA award more personal fouls to players of their opposite race. Chen and Li (2009) show that individuals are not only more altruistic, but also they have less envy towards members of their own groups and when matched with in-group members, are more likely to choose social welfare maximization outcomes. Mitchell et al. (2005) shows that mock jurors tend to decide in favor of individuals who share their ethnicity and were more likely to give longer sentences for other race defendants.

Additionally, there is evidence that individuals evaluate the kindness of a person, taking into consideration how that person has treated other individuals, specially those who are close to them. Fehr and Fischbacher (2004), Engelmann and Fischbacher (2008), Nowak and Sigmund (1998), and Nowak and Sigmund (2005) show that individuals want to help/punish those individuals who are kind/ unkind towards other individuals. Goette et al. (2006) show that individuals who were being trained in different platoons in the Swiss Army had higher levels of 
cooperation and had expected more cooperation from members of the same platoon and when they were given the opportunity to punish defection of third parties punished more harshly if the defection affected a member of their own platoon.

Finally, there is evidence that the kindness or unkindness of individuals creates sympathy or resentment towards all the members of their group and a desire for reciprocity towards them, even those who played no part in the original acts. HughJones and Leroch $(2010,2011)$ show experimentally that individuals who were harmed by an individual reacted by harming other member of that individual's group. Shayo and Zussman (2011) find that judges in Israel treat more favorable members of their own ethnic groups, and this bias increases after a terrorist attack in the vicinity of the court. Edwards et al. (2007) show that consumers in New Zeland and Australia reacted to the French Nuclear Test in the south pacific in the mid1990s firms by reducing their consumption from French firms. Michaels and Zhi (2010) find that American firms' willingness to pay for French inputs decreased after France did not support American efforts to obtain UN Security Council mandate to use military force against Iraq and American imports from France could have being reduced for this reason for as much as $15 \%$.

\section{Model}

Our model is defined for dynamic games with perfect information. $A_{i}$ is the set of strategies (possible mixed) for player $i$ and $A=\prod_{i \in N} A_{i}$ is the set of strategy profiles for all players. $a_{i} \in A_{i}$ is a strategy for individual $i, b_{i j} \in A_{i}$ are the beliefs of individual $i$ regarding the strategy of individual $j$ and $c_{i j k} \in A_{i}$ are the beliefs of individual $i$ about the beliefs of the individual $j$ about the strategy of player $k$ (second order beliefs). Strategy $a_{i}(h)$ is the same as strategy $a_{i}$, but playing history $h$ with probability one. $\pi_{i}: A \rightarrow \mathbb{R}$ are individual $i$ 's "material payoffs". $H$ is the set of the histories of the game and $N$ is the set of the players in the game.

Let $S_{i}$ be a partition of $N$. Partition $S_{i}$ represents the different groups a player can belong according to player $i$. By allowing for different partitions for different players we are allowing for each player to have different beliefs of what the groups are. For example, conceivable, it can happen that a player believes to belong to a group, while others players do not believe it.

We first define what an equitable payoff is and use it as a reference point to evaluate the kindness of a strategy. We only look for the equitable payoffs in the set of efficient strategies. A payoff that is not efficient cannot be equitable, given that the player is giving a lower payoff to the opposing player without any benefit to herself. We define a player's strategy as efficient if there is no other strategy that always gives every player a higher or equal payoff, with strict inequality for at least one player. Because we require that at the equilibria all players maximize at every history, we define our concepts at every history of the game. 


$$
\begin{aligned}
E_{i}=\left\{a_{i} \in A_{i} \mid \text { there exists no } a_{i}^{\prime} \in A_{i} \text { such that for all } h \in H,\left(a_{j}\right)_{j \neq i} \in \prod_{j \neq i} A_{j}\right. \\
\quad \text { and } m \in N, \text { we have that } \pi_{m}\left(a_{i}^{\prime}(h),\left(a_{j}(h)\right)_{j \neq i}\right) \geq \pi_{m}\left(a_{i}(h),\left(a_{j}(h)\right)_{j \neq i}\right), \\
\left.\quad \text { with strict inequality for at least one }\left(h,\left(a_{j}\right)_{j \neq i}, m\right)\right\} .
\end{aligned}
$$

$E_{i}$ is the set of strategies for player $i$ that cannot improve any player at any history without making another player worst off. As the FE and the SRE, we define the equitable payoff as the average of the highest and the lowest of the payoffs in the set of the efficient strategies.

Definition 1 the "equitable payoff" for individual $j$ at history $h$ is given by the following equation:

$$
\pi_{j}^{e}\left(\left(a_{m}(h)\right)_{m \neq i}\right)=\frac{1}{2}\left[\begin{array}{c}
\max \left\{\pi_{j}\left(a_{i}(h),\left(a_{m}(h)\right)_{m \neq i}\right) \mid a_{i} \in A_{i}\right\} \\
+\min \left\{\pi_{j}\left(a_{i}(h),\left(a_{i m}(h)\right)_{m \neq i}\right) \mid a_{i} \in E_{i}\right\}
\end{array}\right] .
$$

We use the equitable payoff as a reference point to evaluate the kindness of a strategy. We propose that a strategy that provides an opponent a higher payoff than the equitable payoff be evaluated as kind and a strategy that provides an opponent with a lower payoff than the equitable payoff be evaluated as unkind. The following function represents how kind an individual believes she is to her opponent.

Definition 2 the kindness of player $i$ towards player $j$ when playing strategy $a_{i}$ is given by:

$$
f_{i j}\left(a_{i}(h),\left(b_{i m}(h)\right)_{m \neq i}\right)=\pi_{j}\left(a_{i}(h),\left(b_{i m}(h)\right)_{m \neq i}\right)-\pi_{j}^{e}\left(\left(b_{i m}(h)\right)_{m \neq i}\right) .
$$

We refer to $f_{i j}$ as the kindness function. We would evaluate the strategy of player $i$ as kind if the kindness function is positive, as the kindness function of player $i$ is positive only when she is sacrificing her own material payoffs to increase the material payoffs of player $j$. The more positive the kindness function is, the higher the material payoff player $i$ is giving to player $j$ and the kinder we would evaluate player $i$ to be. We would evaluate the strategy of player $i$ as unkind if the kindness function is negative as the kindness function of player $i$ is negative only when she is sacrificing her own material payoffs to decrease the material payoffs of player $j$. The more negative the kindness function is, the lower the material payoff player $i$ is giving to player $j$ and the unkinder we would evaluate player $i$ to be.

Now, we define a function that represents how kind a player considers other players behave with their opponents. 
Definition 3 player $i$ 's beliefs about how kind player $j$ is with another player $k$ when she believes she is playing strategy $b_{i j}$ is given by:

$$
\widetilde{f}_{i j k}\left(b_{i j}(h),\left(c_{i j \ell}(h)\right)_{\ell \neq j}\right)=\pi_{k}\left(b_{i j}(h),\left(c_{i j \ell}(h)\right)_{\ell \neq j}\right)-\pi_{k}^{e}\left(\left(c_{i j \ell}(h)\right)_{\ell \neq j}\right) .
$$

$\widetilde{f}_{i j k}$ is similar to $f_{i j}$ but is based on second and third order beliefs. For notational simplicity, we will refer to $f_{i j}\left(a_{i}(h),\left(b_{i m}(h)\right)_{m \neq i}\right)$ as $f_{i j}$,

$$
\widetilde{f}_{i j k}\left(b_{i j}(h),\left(c_{i j \ell}(h)\right)_{\ell \neq j}\right) \text { as } \widetilde{f}_{i j k} \text { and } \pi_{j}\left(a_{i}(h),\left(b_{i m}(h)\right)_{m \neq i}\right) \text { as } \pi_{i} \text {. }
$$

Once we have completed the definitions of kindness and belief as regards kindness we can define an individual's utility function.

Definition 4 The utility of individual $i$ who belongs to group $P \in S_{i}$ is given by:

$$
U_{i}=\pi_{i}+\lambda_{i} \sum_{k \in P \backslash i} \pi_{k}-\theta_{i} \sum_{Q \in S_{i}}\left|\sum_{j \in Q}\left(f_{i j}-\widetilde{f}_{i j i}\right)+\lambda_{i} \sum_{j \in Q} \sum_{k \in P \backslash i}\left(\widetilde{f}_{i j k}-\widetilde{f}_{i k j}\right)\right| .
$$

$\lambda_{i} \in[0,1]$ represents how much player $i$ cares about the other members of her group. At the extremes, if $\lambda_{i}=1$ player $i$ cares as much about the members of her group as she does about herself and if $\lambda_{i}=0$ player $i$ does not care at all about the other members of her group. $\theta_{i}$ is the importance player $i$ gives to reciprocity considerations.

The first term of the utility function: $\pi_{i}$ represents the material payoffs of player $i$. The second term: $\lambda_{i} \sum_{k \in P \backslash i} \pi_{k}$ is the sum of the material payoffs of the players (other than $i$ ) who belong to the same group as player $i$. This term is multiplied by $\lambda_{i}$, which is positive, but equal or lower than one to represent that an individual cares about the payoffs of other members of her group, but (most of the times) not as much as she cares about her own payoffs. From now on, I will refer to the first two terms of the utility function as the "material payoffs" .

The absolute value represents the emotion of reciprocity. Inside the absolute value, there are two terms. The first term: $\sum_{j \in Q}\left(f_{i j}-\widetilde{f}_{i j i}\right)$ represents the direct reciprocity between $i$ and the members of group $Q$. The second term: $\lambda_{i} \sum_{j \in Q} \sum_{k \in P \backslash i}\left(\widetilde{f}_{i j k}-\widetilde{f}_{i k j}\right)$ represents the addition of all the kindness functions between the members of the group of player $i$ and the members of group $Q$. By allowing for the same players with who player $i$ is directly playing to be in this term, we are including the emotion of indirect reciprocity (indirect reciprocity is the emotion of caring not only for how somebody treats us, but how he treats other individuals). However, to avoid further complexity, we limit the emotion of indirect reciprocity to the cases where at least one of the players belong to the same group as player $i$. By including both terms inside the absolute value, we are representing what we call group reciprocity, that when we play with an individual we not only care about how that player treat us, but also how other members of her group treat other members of our own group. From now on, I will refer to this part of the utility function as the "reciprocal payoffs". 
This utility function has four main features. The first one is that the utility of a player increases as the material payoffs of the other members of her group increases. The second one is that the utility of a player increases when she is kind to somebody who is kind to her and when she is unkind to somebody who is unkind to her. The third one is that the utility of a player increases when she is kind to somebody who is kind to a third individual and when she is unkind to somebody who is unkind to a third individual (as long as she belong to her group). Finally, the utility of a player increases when she is kind to somebody who belongs to a group where one member has been kind to a member of her own group and when she is unkind to somebody who belongs to a group where a member has been unkind to a member of her own group.

We use this utility function to define a new equilibrium concept that incorporates the idea that players want to reciprocate when playing against the members of a group in which somebody has been kind or unkind to somebody in their group. We name this equilibrium as sequential group reciprocity equilibrium. As in Geanakoplos et al. (1989), Rabin (1993), and Dufwenberg and Kirchsteiger (2004), in equilibrium, an individual's beliefs have to match both their beliefs regarding beliefs and their actual strategies.

Definition 5 The strategy profile $a \in A$ is a sequential group reciprocity equilibrium if for every $i, j, k \in N$, where $j \neq i, k \neq j$, and for every $h \in H$ it holds the following:

$$
\begin{aligned}
& \text { 1. } a_{i} \in \arg \max _{a_{i}^{\prime}(h) \in A_{i}(h)} U_{i}\left(a \prime_{i}(h),\left(\left(b_{i k}(h)\right)_{k \neq i},\left(c_{i j k}\right)_{k \neq i, j}\right)_{i \neq j}\right) \\
& \text { 2. } b_{i j}=a_{j} \\
& c_{i j k}=a_{k} .
\end{aligned}
$$

From now on, we will refer to the sequential group reciprocity equilibrium as SGRE. The model captures the idea that in the face of acts of kindness or unkindness committed by others, individuals may want to reciprocate not only to those who committed the acts, but also to all of the members of the same group. As a result, the outcomes of different games for individuals who belong to the same groups may be related.

\subsection{Existence}

The FE evaluates the kindness of a player towards another player (for clarity we will refer to both players as "giver" and "receiver") as a function of the payoffs the receiver gets. Because the payoffs of the receiver depend not only on the actions of the giver, but also on her own actions, the FE assumes that a player ("spectator"), who can be the giver or the receiver, evaluates the kindness based on the payoffs the spectator believes the giver wants to give to the receiver. For this, Rabin assumes that the spectator has beliefs not only about the actions of the receiver, but also about the beliefs the giver has about the actions of the receiver). The SRE extends 
the concept of reciprocity for more players, by assuming that the spectator has beliefs about the actions of all other players and about the beliefs of the giver.

Dufwenberg and Kirchsteiger (2004) showed that extending the concept of reciprocity for dynamic games does not allow for a proof of existence using backward induction, given that the best action of each player at each history not only depends on the strategy of the other players, but also on the actions of the same player at other histories. For example, in the Prisoners' Dilemma, shown in Fig. 1 the utility of player 2 at history $D$, depends not only on the strategy of player 1 , but also on her own action at history $C$.

Dufwenberg and Kirchsteiger prove instead the existence of the SRE by maximizing each player's utility at every history in which they play, given not only the strategies of the other players, but also their own actions at other histories. They form a correspondence from the union of the best replies of every player at every history and apply Kakutani's fixed point theorem to prove that this correspondence has a fixed point. This is an equilibrium as no player can improve at any particular history by changing their actions on that history.

Theorem Every finite extensive game with perfect information has a Group Fairness Equilibrium.

The proof of theorem 1 is in the "Appendix".

Our proof of existence follows Dufwenberg and Kirchsteiger (2004) proof of existence of their concept of sequential reciprocity equilibrium by proving that there is a sequential group reciprocity equilibrium where players maximize their utility at every history simultaneously using Kakutani's fixed point theorem.

\section{Sequential Prisoners' Dilemma}

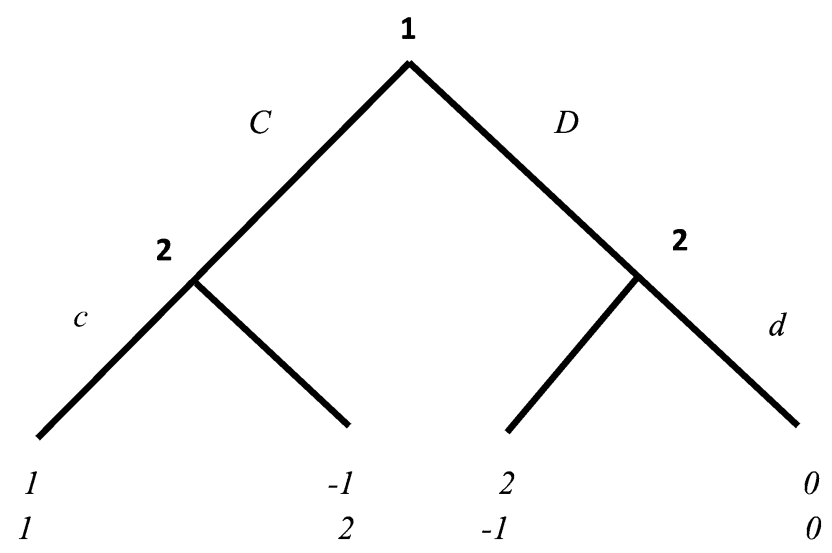

Fig. 1 Sequential prisoners' dilemma 


\subsubsection{Differences with the FE and the SRE.}

There are a few differences between our solution concept and the FE and the SRE.

1. The first difference is that our solution concept includes the concept of SRE, but extends it for reciprocity between the members of different groups. The first term inside the absolute value in our utility function is the equivalent to the utility function used in the SRE. It is in the second term where our concept differs from the SRE by including the emotion of reciprocity for the treatment of other members of our own groups. If, for example, $\lambda_{i}=0$, our solution concept is equivalent to the SRE.

2. The second difference is that our solution concept includes an absolute value instead of the multiplication of the fairness functions, as the FE and the SRE do. The reason for this is that we want to represent that players want to reciprocate a kind or unkind act with the same intensity, unlike the FE and the SRE that make the extreme assumption that players want to reciprocicate a small offense with the highest possible punishment.

3. As the SRE, we do not normalize the reciprocity part of the utility function as the FE does. We do this for simplicity, as our utility function is complicate as it is now. This also allows us to prove the existence of our solution concept, as our utility function is continuous. The utility function of the FE is discontinuous, and therefore it cannot prove its existence.

4. Unlike the FE and the SRE, we include the material payoffs of the other members of the group of each player in the utility function. This allows us to represent that individuals not only want to reciprocate against kind or unkind acts against members of their groups, but also they have the emotion of altruism, especially with members of their own group.

\subsection{Basic results}

While most economists have assumed that players only care about what happens in the games they play, our model assumes that players take into consideration also the interaction of players in other games if they belong to their groups. Our aim is to model how the outcome in one game may affect the outcome of other games.

In this section, we analyze the case of two games with two players in each game and two groups with two members in each group. One of the members of each group plays in each game. To be concise, we will refer to the other member of an individual's own group as her "partner" and the person an individual is playing against as her "opponent".

We analyze the relationship that exists between the outcomes of two seemingly independent games. As Rabin (1993), we study games where the material payoffs may be arbitrarily small or arbitrarily large. For this, we consider a set of games that are exactly the same, with the exception of the scale of their material payoffs. Let $\mathcal{G}$ be the set of games that consists of the set of players $N$, where $N=\{1,2,3,4\}$, the set of strategies $A_{i}$ for all $i \in N$, and the payoff functions $X \cdot \pi_{i}\left(a_{1}, a_{2}\right)$ where 
$i=1,2$ and $Y \cdot \pi_{k}\left(a_{3}, a_{4}\right)$ where $k=3,4$ for $X>0, Y>0$. Let $G(X, Y) \in \mathcal{G}$ be the game that corresponds to the given values $X$ and $Y$.

Given that the material payoffs for each player depend solely on his or her own strategies and those of their opponents, we can think of each game $G(X, Y)$ as a game composed of two materially independent games, one made up of players 1 and 2 and the other made up of players 3 and 4 . Let $\mathcal{G}_{1}$ be the set of games that consists of players 1 and 2 and strategies $A_{1}$ and $A_{2}$, and material payoffs $X \cdot \pi_{1}\left(a_{1}, a_{2}\right)$ and $\left.X \cdot \pi_{2}\left(a_{2}, a_{1}\right)\right)$. Let $G_{1}(X) \in \mathcal{G}_{1}$ be the game that corresponds to a given value of $X$. Let $\mathcal{G}_{2}$ be the set of games that consists of the set of players 3 and 4 , the set of strategies $A_{3}$ and $A_{4}$, and the payoff functions $Y \cdot \pi_{3}\left(a_{3}, a_{4}\right)$ and $Y \cdot \pi_{4}\left(a_{4}, a_{3}\right)$. Let $G_{2}(Y) \in \mathcal{G}_{2}$ be the game corresponding to a given value of $Y$.

Given that a game $G(X, Y)$ is composed of games $G_{1}(X)$ and $G_{2}(Y)$, we sometimes refer to $G(X, Y)$ as "the composite game," to $G_{1}(X)$ as "single game 1", and to $G_{2}(Y)$ as "single game 2 ". We refer to the set of players of single game 1 as $N_{1}$ and to the set of players of single game 2 as $N_{2}$.

Our intention is to identify the outcomes where all players are being kind or unkind. To do so, we rewrite one of the Rabin's definitions. Rabin (1993) works with a single game; hence, his definitions are for a single game only. Rabin defines the outcome of a game as a function of the sign of the kindness function of both players. The outcome of a game is positive if both players are kind to each other and negative if both players are unkind to each other.

Definition 6 For a single game $m$ : (a) an outcome is strictly positive if for all $i, j \in N_{m}, j \neq i$, we have $f_{i j}>0$; (b) an outcome is weakly positive if for all $i, j \in$ $N_{m}, j \neq i$,we have $f_{i j} \geq 0$; (c) an outcome is strictly negative if for all $i, j \in N_{m}, j \neq i$, we have $f_{i j}<0$ and (d) an outcome is weakly negative if for all $i, j \in N_{m}, j \neq i$, we have $f_{i j} \leq 0$.

For some results, we want to allow individuals to be nice to each other if they choose to be. However, this is not possible in every game. For example, in the battle of the sexes, an individual cannot be kind to his or her opponent, given that when a person maximizes their opponent's material payoffs, they are maximizing their own material payoffs as well. We define a game where all individuals can be kind in every possible situation as a kind-game.

Definition 7 A kind-game is a game where $\pi_{i}^{h}\left(a_{i}\right)-\pi_{i}^{l}\left(a_{i}\right)>0$ for every $a_{i} \in A_{i}$ and for every $i \in N$.

A kind-game is a game where all players always have the possibility of being nice to their opponents, as in the Prisoners' Dilemma. This definition excludes games such as the battle of the sexes, where players do not make any sacrifice when they maximize their opponent's material payoffs.

Player 2 (Daughter of family 2) 
Player 1 (Daughter of family 1) Cooperate Defect

\begin{tabular}{l|l|l|}
\cline { 2 - 3 } Cooperate & $4 X, 4 X$ & $0,6 X$ \\
\cline { 2 - 3 } Defect & $6 X, 0$ & $X, X$ \\
\cline { 2 - 3 } & &
\end{tabular}

Game $1 G_{1}(X)$

Player 4 (Mother of family 2)

Player 3 (Mother of family 1)

\begin{tabular}{l|l|l|}
\multicolumn{1}{c}{} & \multicolumn{1}{l}{ Cooperate } & \multicolumn{1}{c}{ Defect } \\
\cline { 2 - 3 } Cooperate & $4 Y, 4 Y$ & $0,6 Y$ \\
\cline { 2 - 3 } Defect & $6 Y, 0$ & $Y, Y$ \\
\cline { 2 - 3 } & &
\end{tabular}

Game $2 G_{2}(Y)$

Example 1 In the next two propositions, we analyze the properties of the sequential group reciprocity equilibria when the material payoffs of a single game change while the material payoffs for the other single game remain the same. In Proposition 1, we analyze the case where $Y$ grows arbitrarily large while $X$ remains constant and in Proposition 2, we analyze the case where $Y$ is small relative to $X$.

Proposition 1 If $\lambda_{i}>0$ and $\theta_{i}>0$ for every $i \in N$ and $G(X, Y)$ is a kind-game, there exists a $\bar{Y}$ for which for all $Y>\bar{Y}$ in the sequential group reciprocity equilibria of $G(X, Y)$ if the sign of the outcome for $G_{2}(Y)$ is strictly positive then the sign of the outcome for $G_{1}(X)$ is strictly positive and if the sign of the outcome for $G_{2}(Y)$ is strictly negative then the sign of the outcome for $G_{1}(X)$ is strictly negative.

All proofs are in the "Appendix".

When the material payoffs of single game 2 grow with respect to those of single game 1, its fairness considerations dominate those of single game 1 if players care at least to some degree about how their partners are treated. If the outcome of single game 2 is strictly negative, the emotions of fairness of every player will be strictly negative and no player would sacrifice their own material payoffs to be nice to their opponent, which would eliminate any strictly positive outcome for game 1 also.

In Example 2, if $Y$ is large enough with respect to $X$ and if the outcome in single game 2 is (defect, defect), which is a strictly negative outcome, then the fairness considerations of single game 2 dominate those of single game 1 , thus eliminating any positive or neutral sequential group reciprocity equilibrium for the composite game and any positive outcome for single game 1 . As the material payoffs of single game 2 grow arbitrarily large, the only outcome for single game 1 in the fairness equilibrium is (defect, defect).

In Example 2, as $Y$ becomes arbitrarily small, individuals in game 2 cooperate only if individuals in game 1 also cooperate and defect if individuals in game 2 defect. As the material payoffs of a single game become arbitrarily small, individuals care more about what is going on in their partner's game and the fairness emotions of the players in the single game with small payoffs is dominated by the 
fairness emotions of their partner's game: players would be kind if their partners are kind and would be unkind if their partners are unkind.

Proposition 2 If $\lambda_{i}>0$ and $\theta_{i}>0$ for every $i \in N$ and $G(X, Y)$ is a kind-game, there exists a $\bar{Y}$ for which for all $Y \in(0, \bar{Y})$ in the sequential group reciprocity equilibria of $G(X, Y)$ if the sign of the outcome for $G_{1}(X)$ is strictly positive then the sign of the outcome for $G_{2}(Y)$ is strictly positive and if the sign of the outcome for $G_{1}(X)$ is strictly negative then the sign of the outcome for $G_{2}(Y)$ is strictly negative.

Sequential group reciprocity equilibria may explain some outcomes that other solution concepts cannot. For example, it is possible that a sequential group reciprocity equilibrium exists where in each Prisoners' Dilemma in Example 2 one player plays "cooperate" while the other plays "defect".

Player 2 (Daughter of family 2)

Player 1 (Daughter of family1)

\begin{tabular}{l|c|c|} 
& \multicolumn{1}{c}{ Cooperate } & Defect \\
\cline { 2 - 3 } Cooperate & 4,1 & $0,3 / 2$ \\
\cline { 2 - 3 } Defect & 6,0 & $1,1 / 4$ \\
\cline { 2 - 3 } & &
\end{tabular}

Player 4 (Mother of family 2)

Player 3 (Mother of family 1)

\begin{tabular}{l|c|c|}
\multicolumn{1}{c}{} & \multicolumn{1}{c}{ Cooperate } & Defect \\
\cline { 2 - 3 } Cooperate & 1,4 & 0,6 \\
\cline { 2 - 3 } Defect & $3 / 2,0$ & $1 / 4,1$ \\
\cline { 2 - 3 } & &
\end{tabular}

Example 2 The payoffs in Example 2 are not symmetrical: in each game one of the players has the opportunity to offset an unkind action carried out by their relative with a much kinder action. If and $\lambda_{i}>0$ and $\theta_{i}>0$ for every player, a sequential group reciprocity equilibrium exists where in single game 1, the daughter of family 1 plays "defect" and the daughter of family 2 plays "cooperate," and where the mother of the family 1 plays "cooperate" and the mother of the family 2 plays "defect". Even if the daughter of family 1 treats the daughter of family 2 badly, overall she would still think well of family 1 given that the mother of family 1 is much kinder than her daughter is unkind. In Example 2, the outcome where the sum of the material payoffs is maximized is one of the sequential group reciprocity equilibria. We generalize the circumstances in which the outcome where the sum of the material payoffs is maximized is a sequential group reciprocity equilibrium. The basic assumption that make this outcome possible is that the payoffs are asymmetric, which means that a player can greatly punish or reward another player with little cost to herself, and players can be nice to each other if they choose to be.

In Example 3, as the value of $X$ increases, the payoffs become more asymmetric. The material payoffs of one player in each game are based on $X$, while the the 
material payoffs of the other player are based on $1 / X$. Let $\mathcal{Q}$ be the set of games that consists of the set of players $N$, the set of strategies $A_{i}$ for each player $i \in N$, and the payoff functions $\left(1 / X \cdot \pi_{1}\left(a_{1}, a_{2}\right), X \cdot \pi_{2}\left(a_{2}, a_{1}\right), X \cdot \pi_{3}\left(a_{3}, a_{4}\right), 1 / X \cdot \pi_{4}\left(a_{4}, a_{3}\right)\right)$ for $X>0$. Let $Q(X) \in \mathcal{Q}$ be the game corresponding to a given value of $X$.

Player 2

Player 1

\begin{tabular}{ll|c|} 
& \multicolumn{1}{c}{ Cooperate } & \multicolumn{1}{c}{ Defect } \\
\cline { 2 - 3 } Cooperate & $4 / X, 4 X$ & $0,6 X$ \\
\cline { 2 - 3 } Defect & $6 / X, 0$ & $1 / X, X$ \\
\cline { 2 - 3 } & &
\end{tabular}

Game 1

Player 3

Player 4

\begin{tabular}{l|l|l|}
\multicolumn{1}{c}{} & \multicolumn{1}{l}{ Cooperate } & \multicolumn{1}{c}{ Defect } \\
\cline { 2 - 3 } Cooperate & $4 X, 4 / X$ & $0,6 / X$ \\
\cline { 2 - 3 } Defect & $6 X, 0$ & $X, 1 / X$ \\
\cline { 2 - 3 } & &
\end{tabular}

Game 2

Example 3 We should note that when $X$ grows arbitrarily large to reach the outcome that maximizes the sum of the material payoffs in a kind-game $Q(X)$, players 1 and 4 must maximize the material payoffs of their opponents, whereas players 3 and 2 must maximize their own material payoffs and, when indifferent, maximize those of their opponents.

Proposition 3 If $Q(X)$ is a kind-game, $\lambda_{i}>0$ and $\theta_{i}>0$, there exists an $\bar{X}$ where for all $X>\bar{X}$ the outcome that maximizes the sum of the payoffs is a sequential group reciprocity equilibrium.

Proposition 3 shows that the outcome where the sum of material payoffs is maximized is a sequential group reciprocity equilibrium as some individuals can greatly help their opponents without incurring a high cost to themselves. By doing so, they can offset the negative emotions set by those individuals that earn high material payoffs by being selfish.

\subsubsection{Group reciprocity over two periods}

Many interesting situations occur in group interactions when individuals anticipate that the kindness or unkindness of their actions will have an effect on the behavior of other individuals. For example, a person may be nice to somebody if she anticipates that their relatives may respond by being nice to her or their relatives. Similarly, terrorists may commit terrible acts to generate negative emotions among the members of opposing ethnic groups to make them fight each other. In this section, we model how individuals may be kind or unkind to influence the behavior 
of other individuals by extending our model of group fairness to the two-period case where single game 1 is played first and then single game 2 and both players in single game 2 observe the outcome of single game 1 before they play. If players in single game 1 know that their actions may affect the outcome of single game 2, they may play differently to influence the actions of the players in single game 2 .

Players in single game 1 maximize their utility in the knowledge that their actions will affect the actions of players in single game 2. Given that they want their partners to be treated kindly in game 2, they may be kind in the first period in order to make their partner's opponent be kind in return, even if they are treated badly. Huck and Lünser (2010) show that in trust games individuals help their partners by sharing more in order to create reputation for their group. ${ }^{2}$ Abbink and Herrmann (2009) create a Vendetta game where members of two groups can reciprocate offences against their own groups. They show that the threat of retaliation decreases the rate of conflict.

Proposition 4 shows that when the payoffs of the composite game are sufficiently asymmetric, the sequential group reciprocity equilibria are outcomes where the sum of the material payoffs are maximized.

Proposition 4 If $Q(X)$ is a kind-game, $\lambda_{i}>0$ and $\theta_{i}>0$ for every $i \in N$, there exists an $\bar{X}$, where for all $X>\bar{X}$, the sequential group reciprocity equilibria in the two-period game are outcomes that maximize the sum of the payoffs.

As $X$ becomes large and the material payoffs become more asymmetric, the emotions of kindness for all players are dominated by the actions of players 1 and 4 (those players that can be very kind or unkind with little cost to themselves). When single game 1 is played first, the actions of player 1 determine the outcome of single game 2 . If player 1 is kind in the first period, player 4 will also be kind in the second period (in contrast, if player 1 is unkind in the first period, player 4 will be unkind in the second period). Since the utility of player 1 is higher if the outcome is positive rather than negative, she will choose to be kind.

Although Proposition 4 appears to contradict the news commonly shown in the media, Fearon and Laitin (1996) show that interethnic cooperation is far more common that interethnic conflict.

Proposition 4 applies to cases where individuals can help others with a low cost to themselves and when other members of their group can repay the kindness the most by being kind to their partners. There are many examples in which friends and relatives of persons with power are often treated nicely in hope of being repaid by them. And there is ample evidence that individuals help others in the moments they need it the most even when they can not repay them in return. In international relations, countries help other countries when there is a humanitarian disaster. Although this may be due to simple altruism, it may also be due to a desire to create goodwill that will be returned later to citizens or firms of the helping country. This is sometimes the case, as some countries give more aid to poor countries with important natural resources to maintain good relations (Lundsgaarde et al. (2010)).

\footnotetext{
2 Tirole (1996) analyze group reputation as the sum of the reputation of its members and how, because individuals past behavior is observed only imperfectly, the reputation of a group is used to estimate the reputation of the members of the group.
} 
In many instances, individuals who can greatly hurt somebody may restraint their actions to avoid retaliation against their relatives later. In situation of asymmetric force, a police officer may restraint from hurting a member of a minority, even if provoked, not because he does not want to hurt him, but because he wants to avoid the violence that this would provoke and may hurt other members from his own group. For example Ron (2000) shows that the Israel army restrained their use of force in the Palestinian Intifada, to avoid generating more violence.

\section{Conclusions}

In this paper, we introduced the emotions of fairness between groups to game theory by extending Dufwenberg and Kirchsteiger's model of sequential reciprocity to groups of individuals. We assumed that individuals have emotions of fairness that include not only the actions played against them, but also against other members of their own group. We defined a new solution concept, which we call "sequential group reciprocity equilibrium" and analyzed how the outcomes of both games are related.

This approach allows us to develop a theoretical model that explains the experimental and empirical evidence that shows that individuals reciprocate not only towards the individuals they are dealing with, but with other members of the same group. We show that for very asymmetric games (those in which some players can greatly punish or reward the opposite player with little cost to themselves), the outcome where the sum of the payoffs is maximized is a SGRE, and if the games are played sequentially, the outcome where the sum of the payoffs is maximized is the only SGRE, as players in the first period want their partners to be treated kindly in period 2, they are kind in order to make their partner's opponent be kind in return.

There are a number of possible extensions to this work. Firstly, in the real world, the majority of interactions are repeated and, therefore, a repeated-game version of group fairness would bring new and more realistic results. In international relations, countries build their relations little by little, increasing their trust through kind actions over time. It is reasonable to think that if an individual or group of individuals are kind or unkind once and again, the feeling of kindness or unkindness will grow larger with repeated interactions. It would be interesting to extend our model by defining a function of kindness that can increase or decrease over time with the repetition of kind or unkind actions.

Secondly, in this paper, we have assumed the value of $\lambda_{i}$ (the importance that a player gives to other members of her group) to be fixed. However, Akerlof and Kranton (2000) show that individuals can, to a certain extent, choose their identity. If individuals can choose how close they are to the groups to which they belong, then they may choose their closeness to a group based on the actions and intentions of the members toward each other and the actions and intentions of other individuals toward the members of the group. For example, if the members of a family are unkind to each other, we should not expect it to be as tightly knit as a family whose members are kind to each other. Furthermore, it has been observed that the existence of a common enemy tends to increase the solidarity among the members 
of a group (Janis 1963). Extending our model by endogenizing the closeness of groups would help to explain many phenomena, such as the increase in religious fervor and nationalism after wars or interethnic conflicts.

Open Access This article is distributed under the terms of the Creative Commons Attribution 4.0 International License (http://creativecommons.org/licenses/by/4.0/), which permits unrestricted use, distribution, and reproduction in any medium, provided you give appropriate credit to the original author(s) and the source, provide a link to the Creative Commons license, and indicate if changes were made.

\section{Appendix}

Proof of existence

Because in equilibrium $a_{j}=b_{i j}, a_{k}=c_{i j k}$ for all $j \neq i, k \neq j$, we write $\left.U_{i}\left(a_{i}(h),\left(b_{i \ell}(h)\right),\left(c_{i \ell k}(h)\right)_{k \neq i}\right)_{\ell \neq i}\right)$ just as $U_{i}\left(a_{i}(h),\left(a_{\ell}(h)\right)_{\ell \neq i},\left(a_{k}(h)\right)_{k \neq i}\right)$.

Note that $U_{i}$ is a continuous function on $a$ as $\pi_{i}, f_{i j}, \widetilde{f}_{i j k}$ and the absolute value are continuous functions. Also note that $U_{i}$ is a quasi-concave function on $a_{i}$ as $\pi_{i}, f_{i j}$, $\widetilde{f}_{i j k}$ are linear in $a$ and the subtraction of the absolute value is a concave function and therefore also quasi-concave.

Let $X_{i}(h)$ be the set of possible actions of player $i$ at history $h$ and let $a_{i}(h) \backslash x$ be the strategy that is exactly the same as strategy $a_{i}$, except at history $h$, where action $x \in X_{i}(h)$ is played.

Let the best response for individual $i$ at history $h$ to be:

$\beta_{i, h}(a)=\arg \max _{x \in X_{i}(h)} U_{i}\left(a_{i}(h) \backslash x,\left(a_{\ell}(h)\right)_{\ell \neq i},\left(a_{k}(h)\right)_{k \neq i}\right)$

As Dufwenberg and Kirchsteiger we define the correspondence $\beta$ as the union of the best responses of every individual at every history

$$
\beta=\prod_{i \in N, h \in H} \beta_{i, h}(a)
$$

Given that $A$ is a nonempty, compact, convex space and $U_{i}$ is continuous in $a$, and quasi-concave in $a_{i}, \beta$ is a nonempty, convex-valued and upper hemicontinuous correspondence.

Therefore, we can apply Kakutani's fixed point theorem. Hence there exists a fixed point in the best response. This fixed point is a Fair Threat Equilibrium as no player can improve by deviating from this strategy.

Proof of Proposition 1 If $\lambda_{i}$ and $\theta_{i}$ are positive for every player, as $Y$ becomes arbitrarily large, the importance of the material payoffs of single game 2 increases and the reciprocal payoffs of player 1 and 2 are dominated by the outcome of single game 2 . Because $G(X, Y)$ is a kind-game, every player can be kind or unkind, but not neutral. As the material payoffs of single game 2 become arbitrarily large and as they cannot change the material payoffs of their partners (since they do not play in the same single game), players 1 and 2 maximize their reciprocal payoffs and are going to be kind if players 3 and 4 are kind and they are going to be unkind if players 3 and 4 are unkind.

Proof of Proposition 2 If $\lambda_{i}$ and $\theta_{i}$ are positive for every player, as $Y$ becomes arbitrarily small, the importance of the material payoffs of single game 2 decreases and the payoffs (both material and reciprocal) of player 3 and 4 are dominated by 
the outcome of single game 1 . Because $G(X, Y)$ is a kind-game, every player can be kind or unkind, but not neutral. As the material payoffs of single game 2 become arbitrarily small and as they cannot change the material payoffs of their partners (since the do not play in the same single game), players 3 and 4 maximize their reciprocal payoffs and are going to be kind if players 1 and 2 are kind and they are going to be unkind if players 1 and 2 are unkind.

Proof of Proposition 3 If $\lambda_{i}$ and $\theta_{i}$ are positive for every player, we will show that when $X$ grows arbitrarily large, in the outcome that maximizes the sum of the material payoffs all players play a best response to each other actions.

Note that in the outcome that maximizes the sum of the material payoffs when $X$ grows arbitrarily large, players 2 and 3 maximize their own material payoffs, and when indifferent, they maximize their opponents material payoffs, while players 1 and 4 maximize the material payoffs of their opponents. Also note that if $X$ grows arbitrarily large in game $Q(X)$, the material payoffs of players 2 and 3 dominate their reciprocal payoffs, while the reciprocal payoffs of players 1 and 4 dominate their material payoffs (as no player can change the material payoffs of their partners since they do not play in the same single game).

Also, if $X$ grows arbitrarily large the payoffs of players 2 and 3 grow arbitrarily large relatively to those of players 1 and 4 and therefore, if players 2 and 3 are treated kindly (by players 1 and 4), not only them, but the other member of their groups would have kind feelings and would want to be kind in return. Because in the outcome that maximizes the sum of the payoffs players 1 and 4 maximize the material payoffs of their opponents and $Q(X)$ is a kind-game, both players are kind.

As $X$ grows arbitrarily large, if players 1 and 4 are kind, they are maximizing their utility by being kind to the players that were kind to their partners, while players 2 and 3 maximize their utility by maximizing their own material payoffs. Therefore, every player is playing their best response in the outcome that maximizes the sum of the material payoffs.

Proof of Proposition 4 If $\lambda_{i}$ and $\theta_{i}$ are positive for every player, when $X$ becomes arbitrarily large, the material payoffs of players 2 and 3 dominate their reciprocal payoffs. However, the actions of player 4 in the second period depend on the actions of player 1 in the first period. As $X$ becomes arbitrarily large the reciprocal payoffs dominate the material payoffs of player 4 . If player 1 kind, player 4 is kind and if player 1 is unkind, player 4 is unkind.

In the first period, as $X$ becomes arbitrarily large the payoffs of player 3 dominate the payoffs of player 1 . Given that the payoffs for player 3 are higher when player 4 is kind than when is unkind, player 1 is going be kind, in order for player 4 to be kind in response. This is a sequential group reciprocity equilibrium as all players are maximizing their utility.

Because players 1 and 4 are maximizing their opponents material payoffs and players 2 and 3 are maximizing their own material payoffs, the group fairness equilibrium of the two-period game is the outcome where the sum of the material payoffs is maximized. 


\section{References}

Abbink K, Herrmann B (2009) Pointless vendettas. Centre for behavioral and experimental social science discussion Paper 09-10

Akerlof GA (1982) Labor contracts as a partial gift exchange. Q J Econ 97:543-569

Akerlof GA, Kranton RE (2000) Economics and identity. Q J Econ 115(3):715-753

Ayres I, Siegelman P (1995) Race and gender discrimination in bargaining for a new car. Am Econ Rev 85(3):304-321

Bolton GE, Ockenfels A (2000) ERC: a theory of equity, reciprocity, and competition. Am Econ Rev 90(1):166-193

Chen Y, Li SX (2009) Group identity and social preferences. Am Econ Rev 99(1):431-457

Dufwenberg M, Kirchsteiger G (2004) A theory of sequential reciprocity. Games Econ Behav 47(2):268-298

Edwards R, Gut A, Mavondo F (2007) Buyer animosity in business to business markets: evidence from the French nuclear tests. Ind Mark Manag 36(4):483-492

Engelmann D, Fischbacher U (2008) Indirect reciprocity and strategic reputation building in an experiment helping game. Games Econ Behav 67(2):399-407

Falk A, Fischbacher U (2006) A theory of reciprocity. Games Econ Behav 54(2):293-315

Fearon JD, Laitin DD (1996) Explaining interethnic cooperation. Am Polit Sci Rev 90(4):715-735

Fehr E, Schmidt K (1999) A theory of fairness, competition, and cooperation. Q J Econ 114(3):817-868

Fehr E, Fischbacher U (2004) Third-party punishment and social norms. Evol Hum Behav 25(2):63-87

Geanakoplos J, Pearce D, Stacchetti E (1989) Psychological games and sequential rationality. Games Econ Behav 1(1):60-79

Goette L, Huffman D, Meier S (2006) The impact of group membership on cooperation and norm enforcement: evidence using random assignment to real social groups. Am Econ Rev 96(2):212-216

Huck S, Lünser G (2010) Group reputations. An experiment foray. J Econ Behav Org 73(2):153-157

Hugh-Jones D, Leroch MA (2010) Group reciprocity. Jena economic research papers 2010 - 066

Hugh-Jones D, Leroch MA (2011) Working Paper. Coventry, UK: Department of Economics, University of Warwick. (CAGE Online Working Paper Series) September 2011 No.52

Janis IL (1963) Group identification under conditions of external danger. $\mathrm{Br} \mathrm{J}$ Med Psychol 36(3):227-238

Kahneman D, Knetsch JL, Thaler RH (1986) Fairness as a constraint on profit seeking: entitlements in market. Am Econ Rev 76:726-741

Krueger AB, Mas A (2004) Strikes, scabs, and tread separations: labor strife and the production of defective bridgestone/firestone tires. J Polit Econ 112(2):253-289

Levine DK (1998) Modeling altruism and spitefulness in experiments. Rev Econ Dyn 1(3):593-622

Lundsgaarde E, Breunig C, Prakash A (2010) Instrumental philanthropy: trade and the geographical distribution of foreign aid. Can J Polit Sci 43(3):733-761

Michaels G, Zhi X (2010) Freedom Fries. Am Econ J Appl Econ 2(3):256-281

Mitchell TL, Haw RM, Pfeifer JE, Meissner CA (2005) Racial bias in Mock Juror decision-making: a meta-analytic review of defendant treatment. Law Hum Behav 29(6):621-637

Nowak MA, Sigmund K (1998) Evolution of indirect reciprocity by image scoring. Nature 393:573-577

Nowak MA, Sigmund K (2005) Evolution of indirect reciprocity. Nature 437:1291-1298

Price J, Wolfers J (2010) Racial discrimination among NBA referees. Q J Econ 125(4):1859-1887

Rabin M (1993) Incorporating fairness into game theory and economics. Am Econ Rev 83(5):1281-1302

Shayo M, Zussman A (2011) Judicial ingroup bias in the shadow of terrorism. Q J Econ 126(3):1447-1484

Tajfel H, Billig MG, Bundy RP, Flament C (1971) Social categorization and intergroup behaviour. Eur J Soc Psychol 1(2):149-178

Tirole J (1996) A theory of collective reputations (with applications to the persistence of corruption and to firm quality). Rev Econ Stud 63:1-22

Zussman A (2013) Ethnic discrimination: lessons from the Israeli online market for used cars. Econ J. doi:10.1111/ecoj.12059 